\title{
Spatial Judgments from Different Vantage Points: A Different Perspective
}

Erik Prytz, Mark Scerbo and Kennedy Rebecca

The self-archived postprint version of this journal article is available at Linköping University Institutional Repository (DiVA):

http:/ / urn.kb.se/ resolve?urn=urn:nbn:se:liu:diva-141764

N.B.: When citing this work, cite the original publication.

Prytz, E., Scerbo, M., Rebecca, K., (2011), Spatial J udgments from Different Vantage Points: A

Different Perspective, Proceedings of the Human Factors and Ergonomics Society Annual Meeting, , 1274-1278. https:// doi.org/ 10.1177/ 1071181311551265

Original publication available at:

https:/ / doi.org/ 10.1177/ 1071181311551265

Copyright: SAGE Publications (UK and US)

http:// www.uk. sagepub.com/home.nav 


\title{
Spatial Judgments from Different Vantage Points: A Different Perspective
}

\author{
Erik Prytz Mark W. Scerbo Rebecca Kennedy \\ Old Dominion University
}

\begin{abstract}
Todorovic (2008) reported that there are systematic errors in the perception of 3-D space when viewing 2-D linear perspective drawings depending on the observer's vantage point. Because Todorovic's findings were restricted to the horizontal plane, the current study was designed to determine whether the magnitude of these errors would be similar in the vertical plane. Participants viewed a 2D image containing rows of columns aligned on parallel converging lines receding to a vanishing point. They were asked to judge where in the physical room the next column should be placed. The results support Todorovic (2008) in that systematic deviations in the spatial judgments depended on vantage point for both the horizontal and vertical planes. However, the pattern of deviation differed between the two planes. While judgments in both planes failed to compensate adequately for the vantage point shift, the vertical plane induced greater distortions of the stimulus image itself within each vantage point.
\end{abstract}

\section{INTRODUCTION}

In many command and control centers and control rooms large computer screens are ubiquitous. They provide an advantage over smaller, more personal, monitors in that multiple observers can look at the same information simultaneously and thus collaborate more easily. However, despite the fact that large screens provide a shared view, the image itself may not be perceived the same. As different observers are located in different positions within the room, their respective viewing angles of the monitor will differ. That is to say, each observer has a unique vantage point. The question then becomes whether this difference in vantage points will affect the spatial judgments of the information depicted in the scene. The current study is the first in a series of experiments that explores this issue.

\section{Vantage Point Distortion of Linear Perspective}

The depiction of 3-dimensional (3-D) space on a 2-D surface must adhere to rules of linear perspective, which convey a projection center formed by parallel converging lines that recede into a vanishing point. In a photograph or painting the projection center is decided by the perspective the artists chooses, but in a computergenerated image it is decided by the designer and the algorithms used for rendering depth. Thus, for every depiction of 3-D space there is a single projection center where the vanishing point corresponds to the artist's vantage point. However, an observer is rarely restricted in space to one vantage point. Thus, researchers have been interested in whether a difference between the observer's vantage point and the projection center causes noticeable distortions in the linear perspective of a perceived scene.

Todorovic (2008) recently investigated how systematic changes in vantage point influenced the perception of 3D-space and evaluated two potential explanations. According to the perspectivetransformation hypothesis, the projection center changes with the observer's vantage point (i.e., the observer perceives a geometrically transformed scene with each shift in vantage point). Studies by Deregowski and Parker $(1988,1992)$ support this hypothesis. They found that when an observer's vantage point shifted laterally from left to right, the perception of objects in a 2-D scene rotated counterclockwise.

By contrast, according to the vantage-point compensation hypothesis perception of perspective is robust and remains invariant with changes in an observer's vantage point (Hagen, 1974; Pirenne, 1970; Shepard, 1992; Vishwanath, Girshick, \& Banks, 2005). However, others have argued that human perception is not able to compensate for at least some distortions (Kubovy 1986; Todorovic, 2005). Although many investigators (Kubovy 1986; Pirenne 1970; Yang \& Kubovy, 1999) agree that changes do occur in the perception of an image from different vantage points, the question is whether this leads to perceptual distortions, and if so under which circumstances they manifest themselves.

Todorovic (2008) attempted to quantify how systematic changes in vantage point influenced the perception of 3-D space from a 2-D linear perspective 
drawing containing rows of columns aligned on parallel lines receding to a vanishing point. He had observers view the image from one vantage point that corresponded with the projection center and two others that deviated along a lateral path. He found that as the vantage point shifted to the right, the perceived location of the columns shifted counter-clockwise supporting the perspective-transformation hypothesis. However, Todorovic was only concerned with the horizontal plane.

Research shows that there are differences in perception along the vertical and horizontal planes (Koffka, 1935); thus, it seems important to investigate the effect of differences in vantage point in the vertical direction. Several studies have shown that the length of an object may be perceived as shorter when viewed along the horizontal axis as compared to the vertical axis. The tendency to underestimate length in the horizontal relative to the vertical dimension has been called the "horizontal-vertical illusion" (Avery \& Day, 1969) and the magnitude of the effect has been reported to be as great as 15\% (Armstrong \& Marks, 1997). Thus, it is possible that differences in vantage point on the perception of 3-D space from 2-D linear perspective images are not equivalent in the horizontal and vertical planes. Thus, one goal of the present study was to replicate the results of Todorovic (2008). In this regard, we expected the projection center to change with the observer's vantage point as predicted by the perspectivetransformation hypothesis. A second goal of the study was to examine the effect of differences in vantage point along the vertical axis. Again, we expected the projection center to change with the observer's vantage point; however, we also expected the magnitude of perspective transformation to differ in the vertical dimension. Specifically, given the tendency to underestimate length in the horizontal dimension as shown in the horizontal-vertical illusion, we expected the effect of different vantage points to be greater for vertical as compared to horizontal judgments.

\section{METHOD}

Participants were seated at three horizontal vantage points (HVPs) and three vertical vantage points (VVPs) and were asked to make spatial judgments based on a stimulus image (see Figure 1). The specific task for the participant was to judge where in the physical room the next column in the depicted colonnades should be placed. The task, stimulus image, and HVPs are replications of Todorovic's (2008) procedure, while the VVPs are novel.

The independent variable was the vantage point of the observer in respect to the image. The dependent variable was the judged position $(\mathrm{cm})$ of the next column in the colonnade.

\section{Participants}

Twelve undergraduate students (10 females and 2 males) from a university population participated in the experiment, with ages ranging from 18 to $27(M=20$, $\mathrm{SD}=2.48$ ). They participated for research credit and all had normal or corrected to normal vision.

\section{Experimental Setup}

The stimulus image was 144 x $107 \mathrm{~cm}$ and backprojected on a large screen. All vantage points were located $133 \mathrm{~cm}$ from the screen, perpendicular to the screen. The first HVP was located directly in front of the image at the projection center. The second and third HVPs were displaced in increments of $133 \mathrm{~cm}$ to the left of the image. Thus, the viewing angles were $0^{\circ}$ (HVP1), $45^{\circ}$ (HVP2) and $64.5^{\circ}$ (HVP3), respectively. The vertical space was constrained by the ceiling and restricted to VVP angles of $0^{\circ}$ (VVP1), $30^{\circ}$ (VVP2), and $45^{\circ}$ (VVP3), respectively.

A plank was positioned halfway $(66.5 \mathrm{~cm})$ between the participants and the screen. A locating rod of the same height as the virtual columns $(107 \mathrm{~cm})$ was held on the plank and the participants then directed the experimenter to move the locating rod right or left until they were satisfied that the rod lined up with the colonnade in question. A tape measure was fixed to the side of the plank facing away from the participant and the placement was recorded in increments of $0.5 \mathrm{~cm}$.

For the VVPs, the locating rod was attached to a vertical post and was flush with the left-most edge of the image. The rod was positioned halfway $(66.5 \mathrm{~cm})$ between the participants and the screen. For the vertical judgments, the experimenter moved the rod up and down as directed by the participant. A measuring tape was fixed to the side facing away from the participants and the placement was recorded in increments of $0.5 \mathrm{~cm}$. 

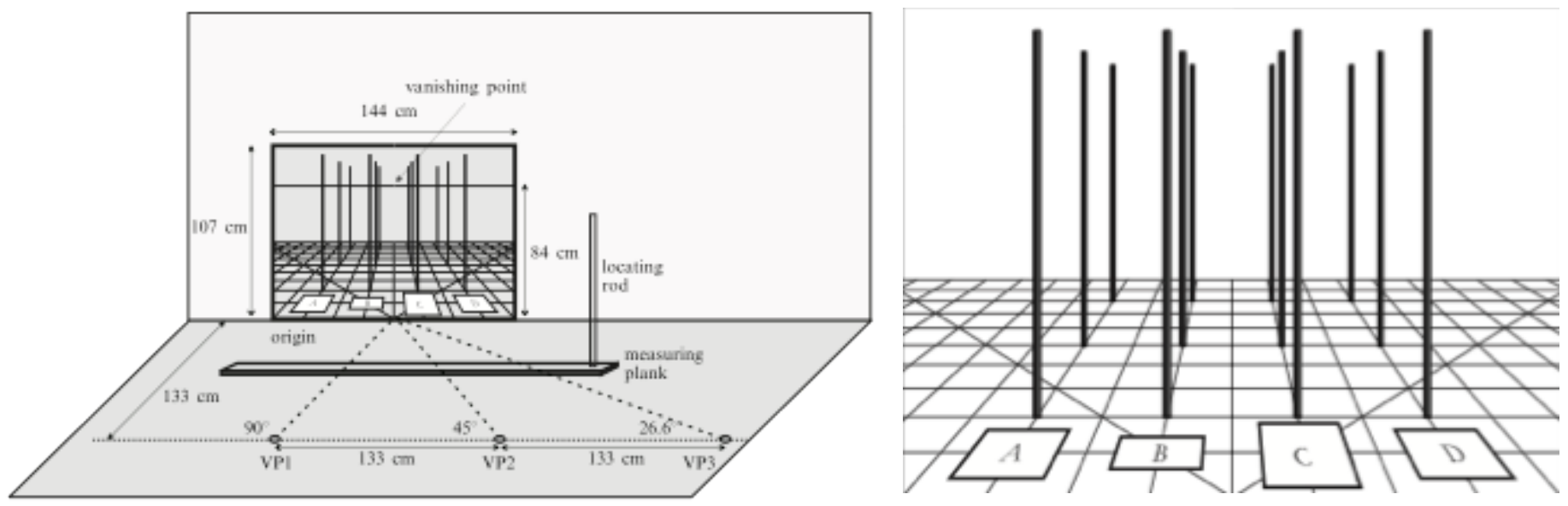

Figure 1. The left figure shows the experimental setup with HVPs, position of the image, and locating rod. The image on the right is the stimulus image adapted for the experiment (from Todorovic, 2008).

\section{Procedure}

The participants were first shown either the image in Figure 1 for the HVPs, or the same image rotated $90^{\circ}$ clockwise for the VVPs. The participants were seated at one vantage point and asked to judge where the next column in one of the four sets of colonnades would be placed in the room between them and the screen. Each colonnade was judged and then the participants were asked to move to a second vantage point. This process was repeated three times, each time with a new vantage point. The participants were instructed to face the opposite way when the image was moved or when switching to a new HVP. For the vertical condition the image was moved rather than the participant, as a safety measure.

The order of vertical or horizontal condition and the three vantage points within each of those conditions were counterbalanced over trials. A partial Latin square with four sequences was used to balance the order of colonnade judgments over all trials.

\section{RESULTS}

The data for analysis were the judgments of offset defined as the measure (in $\mathrm{cm}$ ) of the perceived placement of a column compared to the correct placement, where the correct placement is determined by the continuation of the colonnade into the room along a vector with an angle equal to the viewing angle.

One measure from one participant (the judged position of colonnade $\mathrm{C}$ at the $3^{\text {rd }}$ horizontal vantage point) was deemed to be an outlier ( $>3 \mathrm{SD}$ from the mean) and was replaced by a number 1.5 standard deviations from the mean for that particular measure. No other missing data or outliers were reported.For some statistical procedures the Mauchley's test of sphericity was significant, but as all results hold with both Greenhouse-Geisser and Huynh-Feldt corrections the uncorrected F-strings are reported.

\section{Offset}

A 3 (HVPs) x 4 (Colonnades) repeated measure ANOVA on the offset showed a significant difference between the vantage points, $F(2,22)=130.53, p<.001$, partial $\eta^{2}=0.922$, but not between colonnades, $F(3,33)$ $=.942, p=.43$, partial $\eta^{2}=0.079$, or any interaction, $F(6,66)=.891, p=.507$, partial $\eta^{2}=0.075$.

Table 1. HVP Confidence Intervals

\begin{tabular}{ccccc} 
& & & \multicolumn{2}{c}{$97.5 \%$ CI } \\
\cline { 4 - 5 } HVP & Mean & SD & Lower & Upper \\
\hline HVP 1 & 1.719 & .859 & -0.510 & 3.947 \\
HVP 2 & -27.750 & 2.058 & -33.086 & -22.414 \\
HVP 3 & -53.309 & 4.075 & -63.877 & -42.741
\end{tabular}

As can be seen in the table above, the confidence intervals (CIs) of the three HVPs do not overlap. As the CIs capture the population means with a certainty of $97.5 \%$, they indicate that the judged positions are significantly different at an alpha-corrected, $p<.025$; that is to say, the offsets were successively larger for each HVP. The sign of the number indicates direction, so that a negative number indicates that the average 
placement was to the left of the true position and a positive number that it was to the right.

For the vertical plane, a 3 (VVPs) x 4 (Colonnades) repeated measure ANOVA on the offset showed a significant difference between the vantage points, $F(2$, $22)=55.38, p<.001$, partial $\eta^{2}=0.834$, and between colonnades, $F(3,33)=53.92, p<.001$, partial $\eta^{2}=$ 0.831 , but no interaction, $F(6,66)=1.33, p=.257$, partial $\eta^{2}=0.108$.

Table 2. VVP Confidence Intervals

\begin{tabular}{ccccc} 
& & & \multicolumn{2}{c}{$97.5 \%$ CI } \\
\cline { 4 - 5 } VVP & Mean & SD & Lower & Upper \\
\hline VVP 1 & .385 & .917 & -1.993 & 2.764 \\
VVP 2 & 10.760 & .902 & 8.422 & 13.099 \\
VVP 3 & 22.229 & 2.219 & 16.474 & 27.984
\end{tabular}

Each vantage point is again significantly separated from the others according to the confidence intervals, $p$ $<.025$. Negative numbers indicate that the placement was lower than the correct position, while positive numbers indicate that the participants placed the column higher than the correct position.

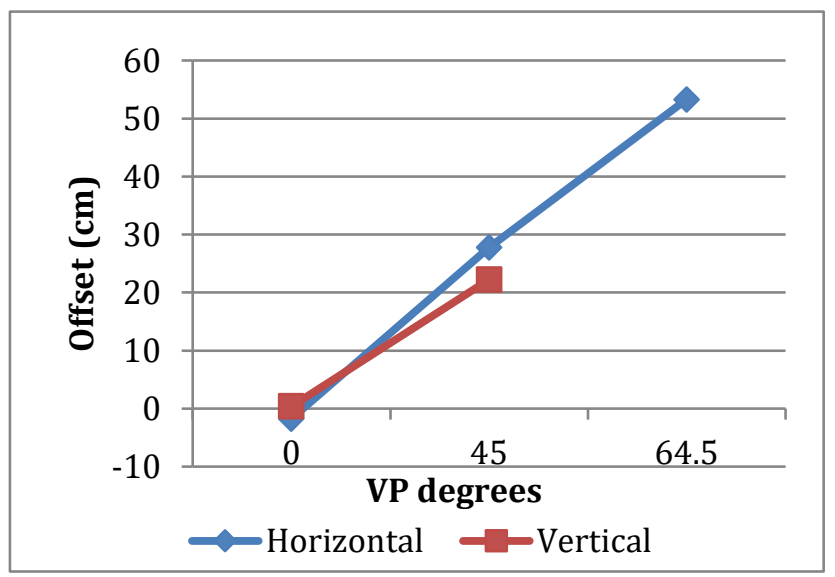

Figure 2. Offset in $\mathrm{cm}$ for $0^{\circ}, 45^{\circ}$ and $64.5^{\circ}$ viewing angle vantage points. Here, positive numbers for the HVPs indicate offset to the left (to illustrate the similarity in offset between horizontal and vertical vantage points).

To examine judgment differences on the horizontal and vertical axes, data were analyzed for the two VPs common to both axes (see Figure 2). A 2 (axis) by 2 (vantage point, $0^{\circ}$ and $45^{\circ}$ ) repeated measures ANOVA showed a significant main effect for vantage point, $F(1,11)=161.783, p<.001$, partial $\eta^{2}=.936$; and an interaction between and VP and axis, $F(1,11)=7.443, p$ $=.020$, partial $\eta^{2}=.404$. However, tests of simple effects showed that the difference between horizontal and vertical judgments at $45^{\circ}$ was not statistically significant, $F(1,11)=4.41, p=.07$.

The difference between the horizontal and vertical conditions lay in the placement of individual colonnades. While the placement was internally consistent for the horizontal plane (see Figure 3), the same does not hold for the vertical plane. That is, for each vertical vantage point the participants had larger offsets for some colonnades than others, as can be seen in Figure 4.

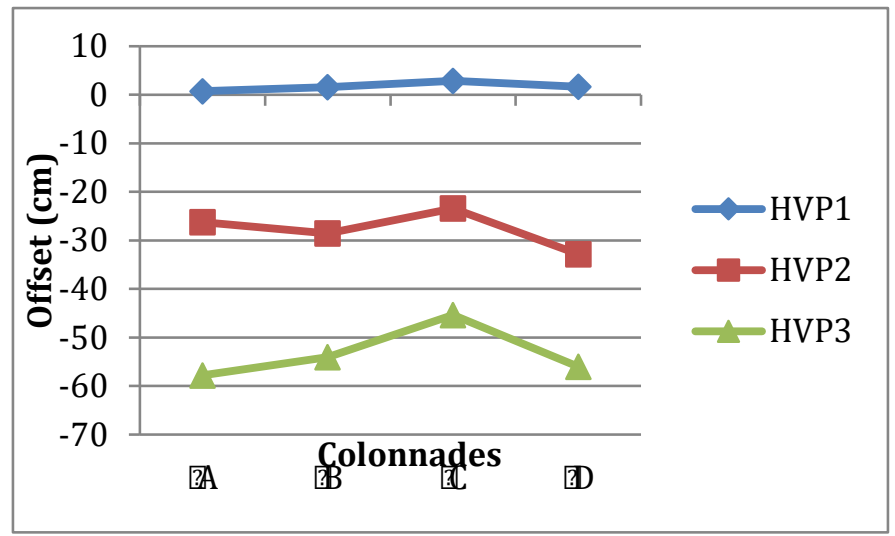

Figure 3. Offset for colonnades A, B, C and D in the horizontal condition.

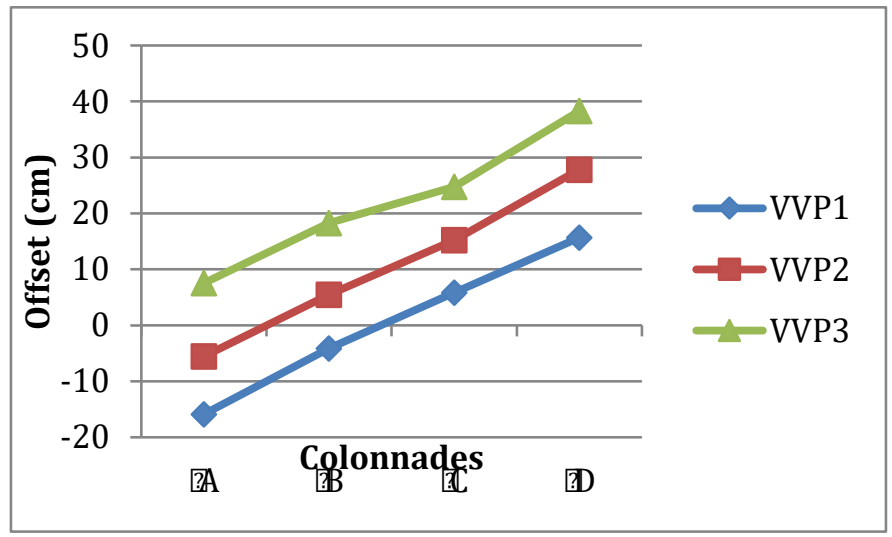

Figure 4. Offset for colonnades A, B, C, and D in the vertical condition.

\section{DISCUSSION}

The results of the present study provide partial support for Todorovic (2008). With respect to the horizontal judgments, our findings provide additional support for the perspective-transformation hypothesis. As the vantage point shifted to the right, the observers perceived the location of the columns to shift in the opposite, counter-clockwise direction. Further, and also consistent with Todorovic, the magnitude of distortion 
increased with greater displacement from the projection center. However, Todorovic found a significant difference among the colonnades and an interaction between vantage points and colonnades. That is, the pattern of the four colonnade directions was perceived differently for each vantage point. This difference may be due to the fact that Todorovic used a betweensubjects design in his study so that different observers judged the colonnades at different vantage points. By contrast, a within-subjects design was used in the present study allowing our participants to render each judgment based on seeing the colonnades at each vantage point.

Regarding the vertical judgments, the findings also support the perspective-transformation hypothesis and again, the more extreme vantage points produced larger offsets. Thus, participants overestimated the position of the correct locations more and more as the height of the image increased.

Contrary to our prediction, based on the verticalhorizontal illusion, there was no statistically significant difference in offset judgments for the horizontal and vertical axes. However, there were still differences in judgments depending on axis. For the vertical plane the colonnades within each vantage point had different offsets (see Figures 3 and 4). It is therefore possible that the horizontal-vertical illusion affected the observers' perception of the stimulus image itself, making the distance between colonnades seem larger than in the horizontal condition. Thus, the pattern of colonnade placements may be indicative of the illusion being present rather than the average offset between the vantage points. Another possible explanation is that these differences may be due to methodological issues. As noted earlier, to create the different VVPs the image was moved, but the participant was moved to create the different HVPs.

\section{Future Works}

Several studies are planned to further investigate the effect of vantage point on spatial judgments. First, a follow-up study is planned to manipulate the image position in the horizontal condition while keeping the participant seated to be consistent with how the image was repositioned in the vertical plane in the present study. Second and more important, a series of experiments is planned to examine the effect of different vantage points on the ability of individuals to interact with objects in the scene in real time.

\section{Conclusions}

The findings of Todorovic (2008) and the current study support the conclusion that as the vantage point shifts from the projection center, the accuracy of an observer's spatial judgments decreases. This is true for both the horizontal and vertical planes, but there are also distinct differences between the two axes. As large screens are common in many different settings today this distortion is particularly relevant, as many observers, each with their own unique vantage point, may each perceive the depicted spatial relationships differently.

\section{REFERENCES}

Armstrong, L., \& Marks, L.E. (1997). Differential effects of stimulus context on perceived length: implications for the horizontal-vertical illusion. Perception \& Psychophysics, 59, 1200-1213.

Avery, G. C., Day, A. H. (1969). Basis of the horizontalvertical illusion, Journal of Experimental Psychology, 81, 376-380.

Deregowski, J.B., \& Parker, D.M. (1988). On a changing perspective illusion withinVermerr's The Music Lesson, Perception, 17, 13-21.

Deregowski, J.B., \& Parker, D.M. (1992). Three-space inference from two-space simulation. Perception \& Psychophysics, 51, 397-403.

Hagen, M. (1974). Picture perception: Toward a theoretical model. Psychological Bulletin, 81, 471-497.

Koffka, K. (1935). Principles of gestalt psychology, London: Routledge \& Kegan Paul.

Kubovy, M. (1986). The Psychology of Perspective and Renaissance Art. Cambridge: Cambridge University Press.

Pirenne, M. H. (1970). Optics, Painting, Photography. Cambridge: Cambridge University Press.

Shepard, R. N. (1992) Mind Sights. New York: W H Freeman.

Todorovic, D. (2005). Geometric and perceptual effects of the location of the observer vantage point for linearperspective images. Perception, 34, 521-544.

Todorovic, D. (2008). Is Pictorial Perception Robust? The Effect of the Observer Vantage Point on the Perceived Depth Structure of Linear-Perspective Images. Perception, 37, 106-125, Pion Limited, London, UK.

Vishwanath D., Girshick A. R., Banks M. S., (2005). Why pictures look right when viewed from the wrong place. Nature Neuroscience, 8, 1401-1410.

Yang. T, \& Kubovy, M. (1999). Weakening the robustness of perspective: Evidence for a modified theory of compensation in picture perception. Perception \& Psychophysics, 61, 456-467. 